\title{
Aspectos clínicos e cirúrgicos da mandibulectomia e maxilectomia no tratamento de patologias orais em cães (Canis familiaris)
}

\section{Clinical and surgical aspects of mandibulectomy and maxillectomy for the treatment of oral patologies in dogs (Canis familiaris)}

\author{
Rosana Pinheiro Botelho, ${ }^{*}$ Marta Fernanda Albuquerque da Silva, ${ }^{*}$ Luciana Gonçalves Pinto, ${ }^{\star *}$ Adelaide Menezes de \\ Magalhães, ${ }^{\star \star \star}$ Alexandre José Alves Lopes, ${ }^{\star \star *}$ Fernando Carteiro***
}

\begin{abstract}
Resumo
Doze cães provenientes do atendimento ambulatorial do Hospital Veterinário do instituto de Veterinária da Universidade Federal Rural do Rio de Janeiro (UFRRJ), foram submetidos a procedimentos de mandibulectomias e/ou maxilectomias totais ou parciais, como forma de resolução de patologias preexistentes na cavidade oral, a saber: fraturas em quatro animais, neoformações mandibulares em seis, e neoplasia maxilar em dois. Os resultados foram avaliados de acordo com os parâmetros de preservação da capacidade de preensão e mastigação, bem como do recolhimento normal da língua. Todos os animais foram capazes de retornar à ingestão de água, após a recuperação anestésica, à alimentação pastosa, em 24 horas, e à ração sólida, dez dias após a cirurgia. Desta forma, constatou-se a melhora da qualidade de vida dos animais ostectomizados, justificando-se a indicação de tais técnicas.
\end{abstract}

Palavras-chave: cão, mandibulectomia, maxilectomia.

\begin{abstract}
Twelve dogs assisted at the Veterinary Hospital of the Universidade Federal Rural do Rio de Janeiro were submited to mandibulectomy or maxilectomy procedures, as a solution to previous oral patologies, such as fractures in four animals, mandibular neoplasms in six animals, and maxilary neoplasms in two animals. The results were analysed considering the competence on prehension, chewing and bringing in the tongue. All dogs were able to drink water just after anesthetic recovery, and to eat soft food 24 hours after surgery. The dry food was accepted in 10 days. Therefore, it was certified the improvement of life quality of all the animals operated, what justifies these techniques indications.
\end{abstract}

Keywords: dog, mandibulectomy, maxillectomy.

\section{Introdução}

É bastante comum a incidência de fraturas na cavidade oral, apresentando a fratura de mandíbula 2,7 a $6 \%$ de todos os casos de fratura em cães (Lantz e Salisbury, 1987, Umphlet e Johnson, 1990, Sgarbosa, 1994 e Kern et al., 1995), sendo também considerada o quarto sítio de localização de câncer em cães (Felizzola, 1994 e Howard, 1994). Segundo as observações de Birchard e Carothers (1990) e Howard (1994), cerca de 5 a $6 \%$ de todas as neoplasias malignas desenvolvem-se nesta área, verificando-se que mais de $50 \%$ dos tumores orais têm características de malignidade (Dobson e White, 1990). O carcinoma de células escamosas ou carcinoma epidermóide abrange 13,4 a $25 \%$ das neoformações; já o epúlides acantomatoso e o tumor de célula basal são responsáveis por 33 a $37 \%$ das neoplasias orais. Os tumores mesenquimais têm uma ordem crescente de ocorrência, representada pelo sarcoma anaplástico $(2 \%)$, osteossarcoma
(5,3 a $14,1 \%)$ e fibrossarcoma (2,3 a 17\%), enquanto 10 a $33 \%$ dos casos descritos são de melanoma maligno. Estes tumores são localmente invasivos, apresentando recidivas freqüentes e podem levar à metástase distante e em linfonodos regionais (Dillon, 1986; Birchard e Carothers, 1990; Schwarz e Withrow, 1990a; Schwarz e Withrow, 1990b; White, 1991; Kosovsky et al., 1991; Salisbury, 1993; Felizzola, 1995; Werner et al., 1997 e Witz et al., 1997).

As técnicas de mandibulectomia e maxilectomia consistem na ressecção de seções variadas da mandíbula e maxila, respectivamente, e ancoramento do segmento ósseo restante nas mucosas e submucosas lingual, labial, bucal ou palatina (Schwarz e Withrow, 1990a; Schwarz e Withrow, 1990b). De acordo com a localização e extensão do comprometimento ósseo mandibular, a técnica é selecionada entre as hemimandibulectomias, que podem ser rostral, central, caudal e total; mandibulectomias bilaterais, que variam entre

\footnotetext{
* Prof a Adjunta do Departamento de Medicina e Cirurgia Veterinária, Instituto de Veterinária, UFRRJ, RJ, Brasil.

** Acadêmica do Curso de Medicina Veterinária, UFRRJ.

*** Médico-veterinário autônomo - RJ, Brasil.
} 
rostral, unilateral total e $3 / 4$ de mandibulectomia. Quando na maxila, seleciona-se dentre pré-maxilectomias unilateral e bilateral; hemimaxilectomia total; maxilectomias central e caudal (Harvey, 1985; Emms, 1987; Salisbury e Lantz, 1988; Schwarz e Withrow, 1990a; Schwarz e Withrow, 1990b; White, 1991; Wallace et al., 1992; Felizzola, 1995; Withrow, 1996; Oliveira, 1996 e Witz et al., 1997).

A massa tumoral deve ser removida com margem de segurança de 1 a $2 \mathrm{~cm}$ em tecido sadio (Harvey, 1985; Salisbury e Lantz, 1988; Schwarz e Withrow, 1990; Kosovsky et al., 1991; Wallace et al., 1992; Salisbury,1993; Withrow, 1996; Witz et al., 1997) na tentativa de evitar a recidiva local.

A associação das técnicas cirúrgicas com a quimioterapia, radioterapia, criocirurgia, imunoterapia ou cirurgia com uso do eletrocautério resulta em prognóstico mais favorável no tratamento das neoplasias, do que a excisão óssea isolada, observando-se maior sobrevida e menor recidiva local (Bostock e Owen, 1975; Harvey, 1985; Greenwood, 1986; Salisbury e Lantz, 1988; Birchard e Carothers, 1990; Frew e Dobson, 1990; Kosovsky, 1991; Schwarz et al., 1991; White, 1991; Oakes et al., 1993; Felizzola et al., 1994 e Witz et al., 1997).

Segundo Kosovsky (1991) e Wallace et al. (1992), o paciente sortador de melanoma apresenta a menor taxa de sobrevida após um ano da cirurgia, quando comparado ao carcinoma epidermóide e ao tumor de células basais. O sexo do animal, o tamanho do tumor e a lise óssea não afetam significativamente a sobrevida, mas sim o tipo de tumor e a idade do paciente.

O presente trabalho tem por objetivo descrever a terapia cirúrgica e procedimentos utilizados no pré e pós-operatório de cães portadores de patologias orais, tais como fraturas cominutivas ou multifragmentadas, fraturas antigas com reabsorção óssea, osteomielite mandibular crônica e neoplasias, submetidos à mandibulectomia e/ou maxilectomia, visando a melhora da qualidade de vida destes animais.

\section{Material e métodos}

No período de novembro/95 a maio/97, foram analisados 12 cães, cinco fêmeas e sete machos, com faixa etária entre dois e 14 anos (Tabela 1). Destes, quatro apresentavam fraturas de mandíbula (Tabela 2), oito eram portadores de neoplasias, sendo duas com localização na maxila (Tabela 3) e seis de origem mandibular (Tabela 4). Todos os procedimentos clínico-cirúrgicos foram realizados no Hospital Veterinário da UFRRJ. Os cães foram catalogados, documentando-se histórico, anamnese, exame físico, medicamentos utilizados, valores referentes ao peso e dados laboratoriais. Nos casos de neoplasia, o tamanho e a localização da massa tumoral, assim como o comprometimento dos linfonodos regionais, foram investigados no exame clínico, e classificados pelo sistema de estagiamento de tumores orais segundo Owen, 1980, apud Schwarz e Witrhow, 1990.

A margem de segurança a ser considerada no transoperatório e o prognóstico do animal foram predeterminados com auxílio de radiografias intra-orais, laterais oblíquas e dorsoventrais, realizadas sob anestesia geral. Nos casos de neoplasia realizaram-se, ainda, radiografias torácicas, sendo os cães por-
Tabela 1 - Relação dos 12 cães com patologias orais, submetidos a ostectomia mandibular ou maxilar, Rio de Janeiro - 1995/1997.

\begin{tabular}{|c|c|c|c|c|}
\hline Animais & Sexo & Idade & Raça & $\begin{array}{c}\text { Patologia } \\
\text { Oral }\end{array}$ \\
\hline 1 & macho & 13 anos & Pequinês & $\begin{array}{c}\text { fratura com } \\
\text { osteólise }\end{array}$ \\
\hline 2 & macho & 15 anos & Pequinês & $\begin{array}{c}\text { fratura com } \\
\text { osteólise }\end{array}$ \\
\hline 3 & fêmea & 4 anos & S.R.D. & $\begin{array}{c}\text { fratura com } \\
\text { osteólise }\end{array}$ \\
\hline 4 & fêmea & 3 anos & S.R.D. & $\begin{array}{c}\text { fratura com } \\
\text { osteólise }\end{array}$ \\
\hline 5 & macho & 6 anos & S.R.D. & $\begin{array}{c}\text { Sarcoma } \\
\text { indiferenciado }\end{array}$ \\
\hline 6 & fêmea & 2 anos & S.R.D. & $\begin{array}{l}\text { Carcinoma } \\
\text { epidermóide }\end{array}$ \\
\hline 7 & macho & 11 anos & Poodle & $\begin{array}{c}\text { Melanoma } \\
\text { amelanótico }\end{array}$ \\
\hline 8 & macho & 14 anos & Doberman & $\begin{array}{c}\text { Melanoma } \\
\text { amelanótico }\end{array}$ \\
\hline 9 & fêmea & 8 anos & $\begin{array}{c}\text { Dogue } \\
\text { Alemão }\end{array}$ & $\begin{array}{c}\text { Melanoma } \\
\text { amelanótico }\end{array}$ \\
\hline 10 & macho & 12 anos & Pequinês & $\begin{array}{c}\text { Melanoma } \\
\text { amelanótico }\end{array}$ \\
\hline 11 & macho & 7 anos & $\begin{array}{l}\text { Pastor } \\
\text { Alemão }\end{array}$ & $\begin{array}{c}\text { Epulides } \\
\text { acantomatoso }\end{array}$ \\
\hline 12 & fêmea & 10 anos & Doberman & $\begin{array}{c}\text { Sarcoma } \\
\text { indiferenciado }\end{array}$ \\
\hline
\end{tabular}

Tabela 2 - Relação dos quatro cães que apresentaram fraturas antigas de mandíbula com reabsorção óssea (osteólise), e as respectivas técnicas de ostectomia aplicadas, Rio de Janeiro - 1995/1997.

\begin{tabular}{c|c|c}
\hline Animais & $\begin{array}{c}\text { Extensão da } \\
\text { osteólise }\end{array}$ & Técnica cirúrgica \\
\hline 1 & $\begin{array}{c}3^{\circ} \mathrm{PM}^{\star} \text { direito ao } 3^{\circ} \\
\mathrm{PM} \text { esquerdo }\end{array}$ & mandibulectomia bilateral rostral \\
\hline 2 & $\begin{array}{c}1^{\circ} \mathrm{M}^{\star *} \text { direito ao } 1^{\circ} \mathrm{M} \\
\text { esquerdo }\end{array}$ & maridibulectomia bilateral rostral \\
\hline 3 & $\begin{array}{c}1^{\circ} \mathrm{PM} \text { ao } 3^{\circ} \mathrm{PM} \\
\text { esquerdos }\end{array}$ & hemimandibulectomia central \\
\hline 4 & $\begin{array}{c}1^{\circ} \mathrm{PM} \text { ao } 3^{\circ} \mathrm{PM} \\
\text { esquerdos }\end{array}$ & hemimandibulectomia central \\
\hline
\end{tabular}

* Dente pré-molar. ${ }^{\star *}$ Dente molar. 
Tabela 3 - Relação dos dois cães que apresentaram neoplasias maxilares e as respectivas técnicas de ostectomia aplicadas, Rio de Janeiro - 1995/1997.

\begin{tabular}{|c|c|c|c|c|c|}
\hline Animais & $\begin{array}{c}\text { Diagnóstico } \\
\text { cito e histopatológico }\end{array}$ & $\begin{array}{l}\text { Estágio clínico do } \\
\text { tumor }\end{array}$ & $\begin{array}{l}\text { Extensão da } \\
\text { osteólise }\end{array}$ & Técnica cirúrgica & $\begin{array}{l}\text { Margem de } \\
\text { segurança }\end{array}$ \\
\hline 5 & Sarcoma indiferenciado & $\begin{array}{c}\text { III } \\
\text { (T3 N2 M0) } \\
\mathrm{N}(+) \\
\end{array}$ & $\begin{array}{c}3^{0} \mathrm{PM}^{\star} \text { ao } \\
2^{\circ} \mathrm{M}^{\star \star} \text { direitos }\end{array}$ & $\begin{array}{l}\text { maxilectomia } \\
\text { central }\end{array}$ & $1 \mathrm{~cm}$ \\
\hline 6 & Carcinoma epidermóide & $\begin{array}{c}\text { II } \\
(\mathrm{T} 2 \mathrm{~N} 1 \mathrm{M} 0) \\
\mathrm{N}(+)\end{array}$ & $\begin{array}{c}\mathrm{C}^{\star \star *} \text { ao } 3^{0} \mathrm{PM} \\
\text { esquerdos }\end{array}$ & $\begin{array}{c}\text { maxilectomia } \\
\text { central }\end{array}$ & $2 \mathrm{~cm}$ \\
\hline
\end{tabular}

* Dente pré-molar.

** Dente molar.

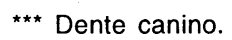

Tabela 4 - Relação dos seis cães que apresentaram neoplasias mandibulares e as respectivas técnicas de ostectomia aplicadas, Rio de Janeiro - 1995/1997.

\begin{tabular}{|c|c|c|c|c|c|}
\hline Animais & $\begin{array}{c}\text { Diagnóstico cito e } \\
\text { histopatológico }\end{array}$ & $\begin{array}{c}\text { Estágio clínico do } \\
\text { tumor }\end{array}$ & $\begin{array}{c}\text { Extensão da } \\
\text { osteólise }\end{array}$ & Técnica cirúrgica & $\begin{array}{l}\text { Margem de } \\
\text { Segurança }\end{array}$ \\
\hline 7 & $\begin{array}{c}\text { Melanoma } \\
\text { amelanótico }\end{array}$ & $\begin{array}{c}\text { III } \\
\text { (T3 NO M0) } \\
\text { N }(-)\end{array}$ & $\begin{array}{c}3^{e} I^{*} \text { direito ao } 1^{2} \mathrm{M} " \\
\text { esquerdo }\end{array}$ & $\begin{array}{l}\text { 3/4 de mandibulecto- } \\
\text { mia }\end{array}$ & $2 \mathrm{~cm}$ \\
\hline 8 & $\begin{array}{c}\text { Melanoma } \\
\text { amelanótico }\end{array}$ & $\begin{array}{c}\| 1 \\
(\mathrm{~T} 2 \mathrm{~N} 1 \mathrm{M} 0) \\
\mathrm{N}(+)\end{array}$ & $\begin{array}{c}2^{0} \mathrm{PM}^{* * *} \text { esquerdo ao } \\
1^{0} \mathrm{PM} \text { direito }\end{array}$ & $\begin{array}{l}\text { mandibulectomia } \\
\text { rostral }\end{array}$ & $2 \mathrm{~cm}$ \\
\hline 9 & $\begin{array}{c}\text { Melanoma } \\
\text { amelanótico }\end{array}$ & $\begin{array}{c}\text { III } \\
\text { (T3 NO MO) } \\
N(-)\end{array}$ & $\begin{array}{l}3^{0} \text { incisivo esquerdo } \\
\text { ao } 2^{0} \text { PM direito }\end{array}$ & $\begin{array}{c}\text { mandibulectomia } \\
\text { rostral }\end{array}$ & $1,5 \mathrm{~cm}$ \\
\hline 10 & $\begin{array}{c}\text { Melanoma } \\
\text { amelanótico }\end{array}$ & $\begin{array}{c}\mathrm{I} \\
(\mathrm{T} 1 \mathrm{NO} \mathrm{M} 0) \\
\mathrm{N}(-)\end{array}$ & $\begin{array}{c}\text { Canino ao } 4^{\circ} \mathrm{PM} \\
\text { direito }\end{array}$ & $\begin{array}{c}\text { hemimandi- } \\
\text { bulectomia horizontal }\end{array}$ & $2 \mathrm{~cm}$ \\
\hline 11 & $\begin{array}{c}\text { Epúlides } \\
\text { acantomatoso }\end{array}$ & $\begin{array}{c}\text { III } \\
\text { (T3 NO MO) } \\
N(-)\end{array}$ & $\begin{array}{l}2^{\circ} \mathrm{PM} \text { direito ao } 2^{\circ} \\
\mathrm{PM} \text { esquerdo }\end{array}$ & $\begin{array}{l}\text { mandibulectomia } \\
\text { rostral }\end{array}$ & $1,5 \mathrm{~cm}$ \\
\hline 12 & Sarcoma anaplástico & $\begin{array}{c}\text { III } \\
(T 3 \mathrm{~N} 2 \mathrm{M0}) \\
\mathrm{N}(+)\end{array}$ & $\begin{array}{c}1^{2} \mathrm{PM} \text { esquerdo ao } \\
2^{0} \mathrm{M} \text { direito }\end{array}$ & $\begin{array}{l}3 / 4 \text { de mandibu- } \\
\text { lectomia }\end{array}$ & $2 \mathrm{~cm}$ \\
\hline
\end{tabular}

* Dente incisivo.

** Dente molar.

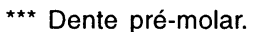

Tabela 5 - Recidiva local, metástases distantes e em linfonodos regionais e sobrevida dos oito cães portadores de neoplasia oral, submetidos à ostectomia (mandibular ou maxilar), Rio de Janeiro - 1995/1997.

\begin{tabular}{|c|c|c|c|c|c|c|}
\hline Animais & $\begin{array}{l}\text { Patologia } \\
\text { Oral }\end{array}$ & Quimioterapia & $\begin{array}{l}\text { Recidiva } \\
\text { local }\end{array}$ & $\begin{array}{l}\text { Metástases } \\
\text { distantes }\end{array}$ & $\begin{array}{c}\text { Metástases em } \\
\text { linfonodos regionais }\end{array}$ & Sobrevida \\
\hline 5 & $\begin{array}{c}\text { Sarcoma } \\
\text { indiferenciado }\end{array}$ & realizada & 5 meses & ausente & ausente & 5 meses \\
\hline 6 & $\begin{array}{c}\text { Carcinoma } \\
\text { epidermóide }\end{array}$ & realizada & ausente & ausente & ausente & 8 meses \\
\hline 7 & $\begin{array}{c}\text { Melanoma } \\
\text { amelanótico }\end{array}$ & realizada & ausente & $\begin{array}{c}\text { lábio } \\
\text { (após } 5 \text { meses) }\end{array}$ & ausente & 6 meses \\
\hline 8 & $\begin{array}{l}\text { Melanoma } \\
\text { amelanótico }\end{array}$ & realizada & ausente & ausente & ausente & 8 meses \\
\hline 9 & $\begin{array}{c}\text { Melanoma } \\
\text { amelanótico }\end{array}$ & realizada & ausente & ausente & ausente & $\begin{array}{l}\text { bom estado geral } \\
\text { há } 12 \text { meses }\end{array}$ \\
\hline 10 & $\begin{array}{c}\text { Melanoma } \\
\text { amelanótico }\end{array}$ & ausente & ausente & ausente & $\begin{array}{l}\text { sub-mandibular ipsilateral } \\
\text { (após } 25 \text { meses) }\end{array}$ & $\begin{array}{c}\text { bom estado geral } \\
\text { há } 12 \text { meses }\end{array}$ \\
\hline 11 & $\begin{array}{c}\text { Epúlides } \\
\text { acantomatoso }\end{array}$ & ausente & ausente & ausente & ausente & $\begin{array}{l}\text { bom estado geral } \\
\text { há } 12 \text { meses }\end{array}$ \\
\hline 12 & $\begin{array}{c}\text { Sarcoma } \\
\text { indiferenciado }\end{array}$ & realizada & ausente & $\begin{array}{c}\text { pulmonar } \\
\text { (após } 1 \text { ano) }\end{array}$ & ausente * & 12 meses \\
\hline
\end{tabular}

* Realizou-se a mandibulectomia e a exérese dos linfonodos submandibulares. 
tadores de metástase pulmonar descartados deste estudo, e biópsias aspirativa e cirúrgica da tumoração, seguidas de análise cito-histopatológica.

Como procedimento pré-operatório, os cães com necrose oral e/ou estomatites severas foram submetidos ao tratamento com metronidazol, ${ }^{1}$ na dosagem de $50 \mathrm{mg} / \mathrm{kg}$, SID, IV, por cinco dias consecutivos.

A pré-anestesia consistiu no uso do maleato de acepromazina, ${ }^{2}$ na dosagem de $0,1 \mathrm{mg} / \mathrm{kg}$, IV, e a indução foi realizada com $10 \mathrm{mg} / \mathrm{kg}$, IV de tiopental sódico. ${ }^{3}$ A manutenção contou com o uso do gás halotano, ${ }^{3}$ em um sistema semifechado, sem absorção.

A tricotomia e a antissepsia foram devidamente efetuadas na região maxilar e mandibular, de acordo com a localização da patologia, com uso de solução de iodo-polivinil-pirrolidona. ${ }^{4}$

A seleção da técnica de mandibulectomia ou maxilectomia utilizada em cada paciente teve por base a localização e extensão do comprometimento ósseo. Dessa forma, procedeuse à incisão dos tecidos moles adjacentes à patologia, e a excisão óssea foi efetuada com auxílio de serra elétrica oscilatória, ${ }^{5}$ irrigando-se o tecido ósseo com solução salina fisiológica. Realizou-se a hemostasia por eletrocoagulação e som cera para osso. ${ }^{6} \mathrm{O}$ padrão de sutura utilizado foi a sutura interrompida simples empregada na mucosa, com fio categute cromado $^{7}$ e na pele, quando incisada, com mononylon. ${ }^{7} \mathrm{~A}$ técnica cirúrgica incluiu a exérese dos linfonodos regionais metastáticos.

A cobertura antibiótica e antiinflamatória no transoperatório contou com a administração de sulfato de gentamicina ${ }^{8}$ (40 $\mathrm{mg} / \mathrm{kg}$ IV) e flunixin meglumine ${ }^{9}(1,1 \mathrm{mg} / \mathrm{kg}, \mathrm{IV})$, que foi mantido durante três dias (IM, SID). Além disso, uma associação de penicilinas $\mathrm{G}$ benzatina, $\mathrm{G}$ procaína e $\mathrm{G}$ potássica cristalina $(40.000 \mathrm{Ul} / \mathrm{kg})$, sulfato de diidroestreptomicina com sulfato de eritromicina ${ }^{10}(20 \mathrm{mg} / \mathrm{kg}) \mathrm{IM}$, e sulfato de gentamicina foi preconizada por sete dias.

Após a recuperação anestésica, ofereceu-se água ao paciente. A ração comercial umedecida (dieta pastosa) foi administrada após 24 horas e mantida até o 10․ dia, quando foi substituída pela alimentação sólida. A limpeza da ferida cirúrgica foi indicada após cada alimentação, utilizando-se a

\footnotetext{
1 Flagyl - Rhodia - Farma Ltda.

2 Thiopental - Cristália.

${ }^{3}$ Fluothane - Zeneca.

${ }^{4}$ Nordiodine - Cinord Sul.

${ }^{5}$ Serra elétrica oscilatória para gesso - Nevoni Equipamentos Odontomédico-hospitalares Ltda., SP.

${ }^{6}$ Cera para Osso - Ethicon.

7 Categute cromado e Mononylon - Cirumédica.

${ }^{8}$ Gentocin - Schering - Plough Veterinária.

${ }^{9}$ Banamine - Schering - Plough Veterinária.

10 Pentabiótico Veterinário - Laboratórios Wyth Ltda. São Bernardo do Campo, SP.
}

solução de iodo-polivil-pirrolidona durante sete dias, e os pontos retirados aos dez dias de pós-operatório. O colar protetor foi utilizado durante este período como meio de prevenção ao autotraumatismo.

Revisões clínicas semanais foram realizadas, nos primeiros trinta dias, para avaliação do restabelecimento das funções de preensão e mastigação dos alimentos. Após esse período, nos animais que apresentavam neoplasias, avaliações clínicas e radiológicas foram indicadas trimestralmente, até completar-se $\circ 12^{\circ}$ mês de pós-operatório, para observação da possibilidade de recidiva local e metástases distantes e/ ou em linfonodos regionais e para o acompanhamento da quimioterapia, quando necessária.

\section{Resultados e discussão}

Diante da não união de fraturas mandibulares, devido à diminuição do suprimento sangüíneo pela artéria mandibular e conseqüente osteólise, as mandibulectomias são indicadas. Os cães (animais no.1 e no.2) com fratura antiga bilateral de corpo mandibular e histórico de fixação óssea através da utilização de pino, apresentaram osteólise em toda a extensão entre as linhas de fratura, realizando-se mandibulectomia bilateral rostral. Os animais $\mathrm{n}^{\circ} 3$ e $\mathrm{n}^{\circ} .4$ apresentaram fratura antiga unilateral ao nível do segundo dente pré-molar e histórico de enxerto ósseo, ocorrendo osteólise nas margens da fratura. Dessa forma, realizou-se a técnica de hemimandibulectomia central, onde o tecido avascularizado foi removido, com imediato alívio da dor, permitindo o retorno às funções de preensão e mastigação.

Os percentuais de incidência dos tumores orais encontrados neste estudo foram de $50 \%$ de melanomas (Figura 1 - animal no.10), 25\% de sarcoma indiferenciado (Figura 2 - animal $n^{0}{ }^{-12}$ ), e $12,5 \%$ de carcinoma de células escamosas (Figura 3 - animal no6) e epulides acantomatoso (Figura 4 - animal no.11). No entanto, Withrow e Holmberg (1983) verificaram um percentual de ocorrência menor de melanoma, embora a incidência de carcinoma de células escamosas tenha sido semelhante ao observado em nosso estudo.

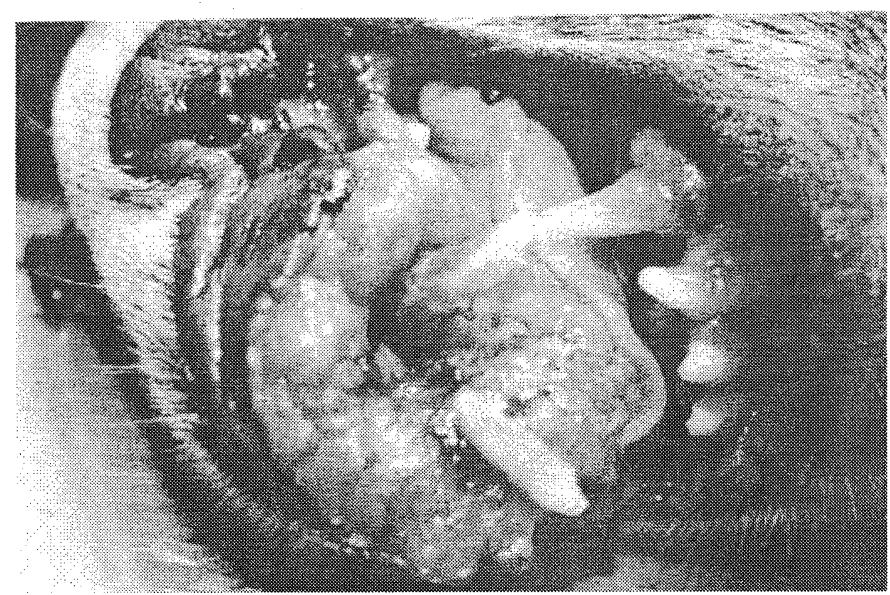

Figura 1 - Melanoma oral (animal $n^{\circ} 9$ ) com estágio clínico do tumor III (T31 NO MO) $\mathrm{N}(+)$ 


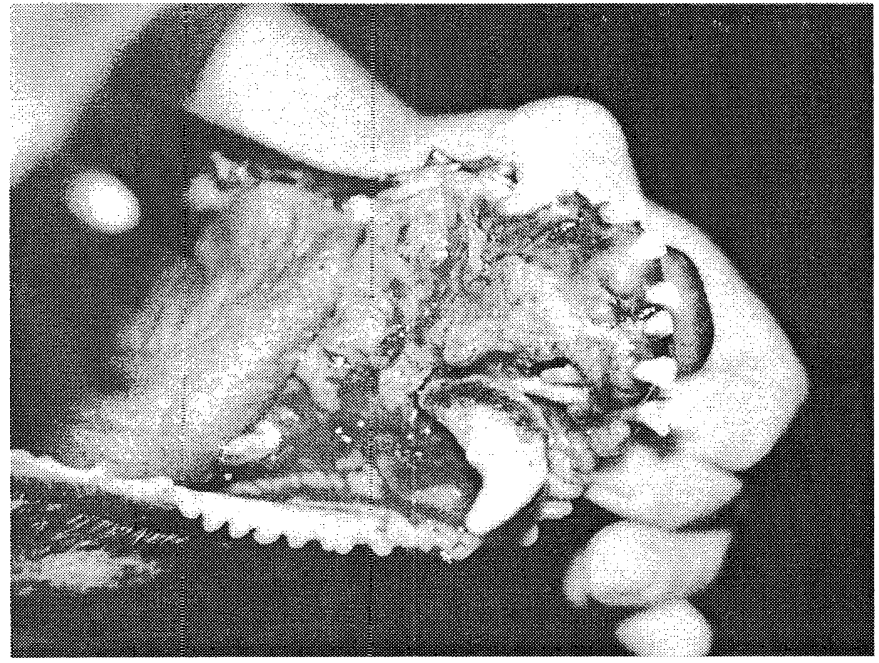

Figura 2 - Sarcoma oral (animal $n^{\circ} 12$ ) com estágio clínico do tumor III (T3 N2 M0) $\mathrm{N}(+)$

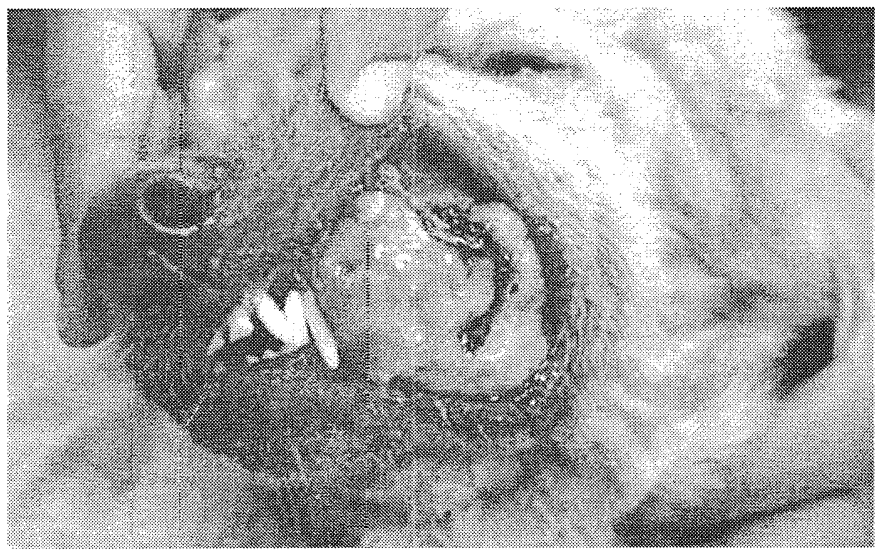

Figura 3 - Carcinoma epidermóide oral (animal no6) com estágio clínico do tumor II (T2 N1 M0) N(+)

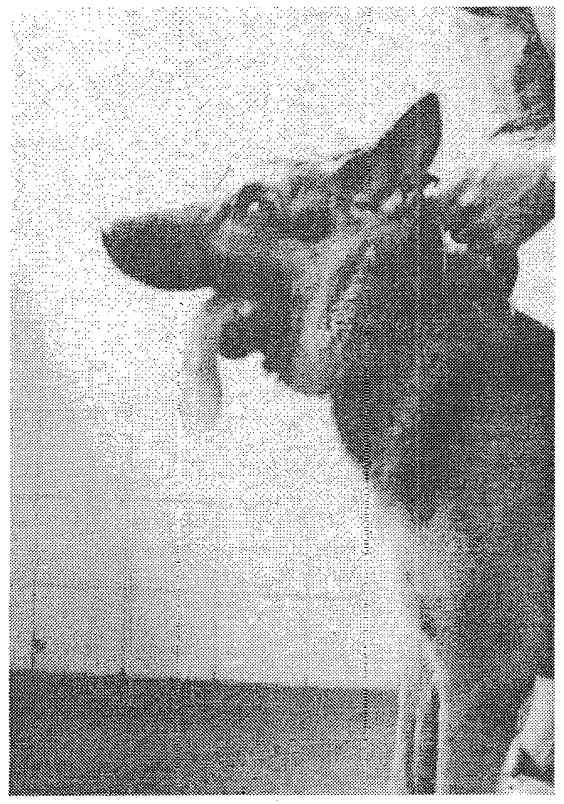

Figura 4 - Animal $\mathrm{n}^{\text {ㅇ }}$ 11 submetido a mandibulectomia rostral devido a presença de epúlides com estágio clínico do tumor III (T3 NO MO) N(-)
O percentual de raças acometidas por neoplasia foi de $25 \%$ em Doberman e S.R.D. e de 12,5\% em Dogue Alemão, Pastor Alemão, Poodle e Pequinês, com idades variando entre dois e 14 anos. Observou-se que esta ampla faixa etária, incluindo animais adultos jovens, foi semelhante aos resultados encontrados por White e Gorman (1989), Kosovsky et al. (1991) e Felizzola (1995), porém Oakes et al. (1993) verificaram a ocorrência apenas entre sete e 11 anos.

Lantz e Salisbury (1987), Salisbury e Lantz (1988), Schwarz e Withrow (1990a) e Salisbury (1993) utilizaram fios absorviveis de longa duração, como poliglactina 910 (trançado), polidioxanone e polipropileno (monofilamentosos), evitando adesão de alimentos e formação de úlceras na cavidade oral. Barateando o custo de material empregado, utilizou-se, neste estudo, fio inabsorvível monofilamentoso, obtendo-se também resultado satisfatório, sem ocorrência destas complicações. Houve cicatrização em 11 dos 12 cães $(91,67 \%)$ ostectomizados. O animal $\mathrm{n}-5$, submetido à maxilectomia central, apresentou deiscência parcial da sutura, mas alimentou-se sem aspiração de água ou de alimento, havendo cicatrização por segunda intenção.

$\mathrm{Na}$ excisão tumoral foi possível respeitar margem de segurança de 1 a $2 \mathrm{~cm}$, entre o tumor e o tecido sadio, em todos os animais portadores de neoplasias (Tabelas 3 e 4), na tentativa de controlar a recidiva local, conforme sugerem Harvey (1985), White e Gorman (1989), Birchard e Carothers (1990), Dobson e White (1990), Schwarz e Withrow (1990a), Kosovsky et al. (1991), Wallaco et al. (1992), Harvey e Emily (1993) e Salisbury (1993). Pôde-se observar, com as avaliações clínicas e radiológicas, que, ao respeitar-se esta margem de segurança, a recidiva local ocorreu apenas no animal $n^{\circ} 5$.

White e Gorman (1989), Sgarbosa e lamaguti (1994) e Kudo et al. (1994) indicam estabilização da mandíbula para evitar o deslocamento do ramo mandibular, conseqüente maloclusão e formação de úlcera no pálato, necessitando de extração do canino. Harvey (1985), Schwarz e Withrow (1990a) e Bichard e Carothers (1990), contra-indicam a utilização desta prótese devido a sua migração e/ou rejeição. Neste estudo, não se estabilizou a mandíbula através da utilização de próteses, e somente os animais submetidos a $3 / 4$ de mandibulectomia (animais $\mathrm{n}^{\circ} 7$ e $\mathrm{n}$ ㅇ 12), e à hemimandibulectomia horizontal (animal no 10) apresentaram deslocamento do ramo mandibular contralateral, mas sem a necessidade de extração do dente canino, pois não houve formação de úlcera no palato durante os 12 meses de observação.

Segundo Salisbury (1993), não se justifica uma excisão completa da mandíbula, caudal ao $3^{\circ} \mathrm{PM}$ ou 4 $\mathrm{PM}$, e de acordo com Schwarz e Withiow (1990), posterior ao $2^{\circ} \mathrm{PM}$, pois compromete a musculatura sublingual, havendo queda da língua e perda da função de preensão, e o animal poderá restabelecer o controle apenas parcial da língua, e depois de algum tempo. No entanto, sete $(87,5 \%)$ dos oito animais com intervenção na mandíbula neste estudo, foram submetidos à mandibulectomia caudal ao $3^{\circ} \mathrm{PM}$, sem prejuízo à preensão e mastigação do alimento, uma vez que se reinseriu a musculatura sublingual.

Schwarz e Withrow, 1990a, Schwarz e Withrow, 1990b, Kosovsky et al. (1991), Wallace et al. (1992) e Salisbury (1993) ofereceram água 24 horas após a cirurgia, mas todos os animais deste estudo foram capazes de retornar à ingestão 
de água imediatamente após a recuperação anestésica, sendo desnecessário aguardar um maior período de tempo.

Witz et al. (1997) forneceram alimentação líquida após 24 horas e pastosa apenas na segunda semana, enquanto os animais do estudo de Kosovsky et al. (1991) receberam dieta após 24 horas, mas só retornaram à alimentação oral após três dias. Todos os animais deste estudo mantiveram a função e o recolhimento normais da língua, a capacidade de preensão e de mastigação, sendo capazes de ingerir alimento pastoso 24 horas após a cirurgia, assim como relatado por Greenwood (1986), Salisbury e Lantz (1988), Schwarz e Withrow (1990), Wallace et al. (1992) e Salisbury (1993), dispensando as técnicas de alimentação enteral por sonda nasoesofágica, por faringostomia ou por gastrostomia. Harvey (1985) fez uma adaptação da dieta pastosa à sólida entre o $10^{\circ}$ e $14^{\circ}$ dias, e Salisbury (1993) só iniciou no $30^{\circ}$ dia de pós-operatório; no entanto, todos os animais deste estudo retornaram à alimentação sólida no $10^{\circ}$ dia após a cirurgia, sem nenhum prejuízo à cicatrização dos tecidos adjacentes.

Segundo White e Gorman (1989), Bichard e Carothers (1990), Schwarz e Withrow (1990), Schwarz et al. (1991), Salisbury (1991), Kosovsky et al. (1991), Wallace et al. (1992), Salisbury (1993) e Witz et al. (1997) existem outras complicações inerentes à mandibulectomia e maxilectomia, como o problema estético, a rânula e a osteomielite. Neste trabalho, acreditamos que, devido aos cuidados transoperatórios e da terapia

\section{Referências}

BIRCHARD, S., CAROTHERS, M. Agressive surgery in the management of oral neoplasia. Vet Clin North America: Small Animal Pratice v. 20 n. 4, p. 1117-1140, 1990.

DILLON, A. R. Cap. 1: The oral cavity, p. 1-53. In: JONES, B. D., Canine and feline gastroenterology, W. B. Saunders Company, 1986.

DOBSON, J. M., WHITE, R. A. S. Oral tumours in dogs and cats. In: Practice, v. 12, n. 4, p. 136-146, 1990.

FELIZZOLA, C. R. Estudo comparativo de neoplasias bucais em cães - Avaliação dos aspectos clínicos, da histopatologia e citologia esfoliativa. 80 p. Dissertação (Mestrado) - Fac de Med Vet Zootec. Univ. SP. 1995.

FREW, D. G., DOBSON, J. M. Tumors of the mandible and maxilla in dogs. Veterinary Annual, v. 31, p. 193-203, 1990.

GREENWOOD, K. M. Conduta cirúrgica em certas neoplasias da cavidade oral dos cães, p. 132-138. In: BOJRAB, M. J., Cirurgia dos pequenos animais, 1986.

HARVEY, C. E. Oral surgery, p. 156-180. In: Vet Dentistry, Philadelphia: Saunders, 1985.

, EMILY,P. P. Oral surgery, p. 312-377. In: Small animal dentistry., Baltimore: Mosby, 1993.

KOSOVSKY, J. K., MATTHIESEN, D. T., MARRETTA, S. M., PATNAIK, A. $K$. Results of partial mandibulectomy for the treatment of oral tumors in 142 dogs. Vet Surgery, v. 20, n. 6, p. 397-401, 1991.

KUDO, T., TAKEUCHI, S., YAMAZOE, K., MARUYAMA, Y. Bioceramic implantation in the intermandibular space in bilateral rostral mandibulectomy of the dog. J Vet Med Science v. 56, n. 1, p. 115-119, 1994.

LANTZ, G. C., SALISBURY, S. K. Partial mandibulectomy for treatment of mandiular fractures in dogs: eight cases (1981-1984). JA VMA, v. 191, n. 2, p. 243-245, 1987.

OAKES, M. G., LEWIS, D. D., HEDLUND, C. S., HOSGOOD, G. Canine oral neoplasia. The Compendium collectin, v. 15, n. 1, p. 90-104, 1993. antibacteriana empregada, não ocorreu osteomielite. Rânula ocorreu no animal $\mathrm{n}^{\circ} 10$, que foi submetido à hemimandibulectomia horizontal, regredindo apenas com a medicação do protocolo, em 72 horas. Houve melhora da qualidade de vida em todos os casos de mandibulectomia e maxilectomia.

De acordo com o protocolo dos trabalhos de Dillon (1986), Greenwood (1986), Schwarz e Withrow (1990), Schwarz et al. (1991), Kosovsky et al. (1991), White (1991), Wallace et al. (1992) e Salisbury (1993) realizaram-se avaliações clínicas e radiológicas durante 12 meses após a cirurgia, constatandose a ocorrência de metástases distantes nos animais $n^{\circ} 7$ e no 12, e metástase em linfonodo regional no animal no 10 (Tabela 5).

De acordo com o protocolo de estabelecido por Harvey, 1985; Salisbury e Lantz, 1988; Birchard e Carothers, 1990; Frew e Dobson, 1990; Kosovsky, 1991; Schwarz et al., 1991; White, 1991; Oakes et al., 1993; Felizzola et al., 1994 e Witz et al., 1997 utilizou-se eletrocautério no transoperatório, objetivando maior sobrevida e menor recidiva local. Segundo Kosovsky (1991) e Wallace et al. (1992), o paciente portador de melanoma apresenta a menor taxa de sobrevida após um ano da cirurgia (21 a 27\%), com média de 9,1 meses, comparado ao carcinoma epidermóide com média de 19,2 meses. No entanto, neste estudo observou-se que a sobrevida dos animais com melanoma, após um ano da cirurgia, foi de $50 \%$, com média de 9,5 meses, enquanto a dos portadores de carcinoma epidermóide foi de apenas 5 meses (Tabela 5).

SALISBURY, S. K. Problems and complications associated with maxillectomy, mandibulectomy, and oronasal fistula. Problems in Vet Med, v. 3, n. 2, p. 153-169, 1991.

. Maxillectomy and mandibulectomy, p. 521-529. In: SLATTER, D., Textbook of small animal surgery. Vol. 1, 2nd ed. Philadelphia: Saunders, 1993.

, LANTZ, G. C. Long-term results of partial mandibulectomy for treatment of oral tumors in 30 dogs. J Am An Hosp Assoc, v. 24, p. 285-294, 1988.

SCHWARZ, P. D., WITRHOW, S. J. Mandibulectomy, p. 850-861. In: BONE, D. L., BOJRAB, M. J. Current techniques in small an surg, 3nd ed., Philadelphia: Lea \& Febiger, 1990a.

SCHWARZ, P.D., WITRHOW S. J.. Maxillectomy and premaxillectomy, p. 861-870. In: BONE, D. L., BOJRAB, M. Current techniques in small an surg, 3nd ed., Philadelphia: Lea \& Febiger, $1990 \mathrm{~b}$.

SCHWARZ,P.D., WITHROW, S.J., CURTIS, C. R., POWER, B. E., STRAW, R. C. Mandibular resection as a treatment for oral cancer in 81 dogs. $J$ Am An Hosp Assoc, v. 27, p. 601-610, 1991.

SGARBOSA, S. H. P. V., IAMAGUTI, P. Fixação de sínfise da mandíbula em cães pós mandibulectomia parcial rostreal: avaliação histologica e radiográfica. CONG BRAS CIR ANES VET, Curitiba, Anais... p. 6-10, nov. 1994.

WALLACE, J., MATTHIESEN, D. T., PATNAIK, A. K. Hemimaxillectomy for the treatment of oral tumors in 69 dogs. Vet Surg, v. 5, n. 21, p. 337341, 1992.

WHITE, R. A. S. Mandibulectomy and maxillectomy in the dog: long term survival in 100 cases. J of Small An Pratice, v. 32, n. 2, p. 69-74, 1991. GORMAN, R. T. Wide local excision of acanthomatos epulides in the dog. Vet Surg, v. 18, n. 1, p. 12-14, 1989.

WITHROW, S. J., HOLMBERG, D. L. Mandibulectomy in the treatment of oral cancer. J Am An Hosp Assoc, v. 19, p. 273-286, 1983.

WITZ, M. I., RAISER, A. G., CONTESINI, E. A. Pré-maxilectomia em cães (Canis familiaris Linnaeus, 1758). Rev Bras Ciên Vet, v. 4, n. 1, p. 3134, 1997. 